\title{
ANTIBIOTIC RESISTANCE PATTERN IN PSEUDOMONAS AERUGINOSA SPECIES ISOLATED AT INDORE (M.P.)
}

\author{
Prafulla Songara ${ }^{1}$, Sushma Neema², Vinita Kothari ${ }^{3}$, Ruchi Sachdeva ${ }^{4}$, Rupal Deva ${ }^{5}$
}

\section{HOW TO CITE THIS ARTICLE:}

Prafulla Songara, Sushma Neema, Vinita Kothari, Ruchi Sachdeva, Rupal Deva."Antibiotic Resistance Pattern in Pseudomonas Aeruginosa Species Isolated at Indore (M.P.)". Journal of Evolution of Medical and Dental Sciences 2014; Vol. 3, Issue 01, January 06; Page: 109-114.

ABSTRACT: INTRODUCTION: Pseudomonas aeruginosa is an aerobic, motile, gram negative rod that belongs to the family, pseudomonadaceae ${ }^{2}$. Its general resistance is due to a combination of factors ${ }^{3}$.Regional variations in the antibiotic resistance exist for different organisms, including $\mathrm{P}$. aeruginosa and this may be related to the difference in the antibiotic prescribing habits. So, we aimed in the present study, to determine the status of antimicrobial resistance to antipseudomonadal agents and the magnitude of the multidrug resistance in these organisms.MATERIALS AND METHODS: This study was conducted during $1^{\text {st }}$ January 2013 to $30^{\text {th }}$ September 2013. During this period total of 5877 samples were tested, out of 5877 samples, 1693 samples showed growth on culture and out of 1693 samples, 152 Pseudomonas aeruginosa were isolated. Identification \& sensitivity of all isolates were done by BD Phoenix ${ }^{\mathrm{TM}}$ Automated Microbiological System. The antibiotics which were included in the panel were ciprofloxacin, levofloxacin, gentamicin, amikacin, tobramycin, aztreonam, ceftazidime, cefepime, piperacillin, piperacillin/tazobactam, ticarcillin/tazobactam, imipenem, meropenem and colistinaccording to CLSIs guidelines.RESULT: In the present study, the highest numbers of Pseudomonas infections was found in pus followed by urine and Endotracheal secretion. Pseudomonas aeruginosa isolated from various samples were resistant to aztreonam, ciprofloxacin followed by levofloxacin, ceftazidime, cefepime, amikacin, imipenem \& colistin.CONCLUSION: To prevent the spread of the resistant bacteria, it is critically important to have strict antibiotic policies wherein surveillance programmes for multidrug resistant organisms and infection control procedures need to be implemented.

INTRODUCTION: Antibiotic when first introduced was considered as a magic bullet. A single injection of penicillin could eradicate a life threatening infection. Unfortunately with time due to malpractices, most of the cheaper antibiotics have lost their efficacy and more and more expensive and complicated antibiotics were introduced and marketed to combat simple infection ${ }^{1}$. The microbial pathogens, as well as, their antibiotic sensitivity pattern, may change from time to time and place to place. Therefore knowledge of current drug resistance pattern of the common pathogenic bacteria in a particular region is useful in clinical practice.

Pseudomonas aeruginosa is an aerobic, motile, gram negative rod that belongs to the family, pseudomonadaceae ${ }^{2}$. Its general resistance is due to a combination of factors ${ }^{3}$. It is intrinsically resistant to antimicrobial agents, due to the low permeability of its cell wall. It has the genetic capacity to express a wide repertoire of resistance mechanisms. It can become resistant through mutations in the chromosomal genes which regulate the resistance genes. It can acquire additional resistance genes from other organisms via plasmids, transposons and bacteriophages. In recent years, a considerable increase in the prevalence of multidrug resistance (MDR) in P. aeruginosa has been noticed, leading to high morbidity and mortality 4,5 . 


\section{ORIGINAL ARTICLE}

Regional variations in the antibiotic resistance exist for different organisms, including P. aeruginosa and this may be related to the difference in the antibiotic prescribing habits. Periodic testing and analysis of antibiotic resistance would enable the physicians to detect the trends in the resistance pattern to the commonly prescribed antibiotics in a given organism. So, our aim in this study was to determine the status of antimicrobial resistance to anti-pseudomonadal agents and the magnitude of the multidrug resistance in these organisms.

MATERIALS \& METHODS: This study was conducted at the Central lab, Indore (M.P.), which is NABL accredited lab. The present study comprised of 152 Pseudomonas aeruginosa positive samples from period of $1^{\text {st }}$ January 2013 to $30^{\text {th }}$ September 2013 which varied fromurine, sputum, pus, pleural fluid, BAL, ascitic fluid, blood etc. samples submitted for microbiological diagnosis from various hospitals in Indore \& its surrounding region.

Sample processing-The samples were selected on the basis of their growth on routine Blood agar \& MacConkey medium which showed lactose non-fermenting pale colonies which were oxidase test positiveIdentification \& sensitivity of all isolates were done by BD Phoenix ${ }^{\mathrm{TM}}$ Automated Microbiological System. The antibiotics which were included in the panel were ciprofloxacin, levofloxacin, gentamicin, amikacin, tobramycin, aztreonam, ceftazidime, cefepime, piperacillin, piperacillin/tazobactam, ticarcillin/tazobactam, imipenem, meropenem and colistin according to CLSIs guidelines.

RESULTS: A total of 5877 samples were tested. Out of 5877 samples, 1693 samples showed growth on culture and out of 1693 samples, 152 Pseudomonas aeruginosa, 8 Pseudomonas putida \& 3 Pseudomonas fluorescence were isolated and tested for antibiotic sensitivity.

DISCUSSION: Pseudomonas aeruginosa is a major cause of nosocomial infection. It is one of the important causes of morbidity among hospitalized patients. Despite advances in sanitation facilities and the introduction of a wide variety of antimicrobial agents with anti-pseudomonadal activities, life threatening infections caused by Pseudomonas aeruginosa continue to be hospital infections.

Critical factors in the survival of Pseudomonas aeruginosa in unfavorable environments include its extreme ability to transform from a mobile "swarmer" cell to a glycocalyx enclosed microcolony which serves to protect the organisms against the active phagocytes, surfactants, enzymes and high levels of specific antibodies and its ability to survive and multiply even with minimum nutrients. Nowadays, the prevalence of Pseudomonas aeruginosa and the new resistant strains continue in both community-acquired pathogens and hospital acquired infections ${ }^{6}$.

As Pseudomonas aeruginosa causes serious infections, and is one of the leading causes of hospital acquired infections, several studies have been carried out to detect antibiotic sensitivity pattern for the various drugs available. Such study helps clinicians for the better management of patients. So the present study was conducted to determine the antibiotic sensitivity pattern of Pseudomonas aeruginosa isolated from various clinical samples in central India.

In the present study the isolation rate of Pseudomonas aeruginosa was comparable with other studies ${ }^{7}$. The distribution of the isolates is significantly affected by the type of hospital from which they have been isolated. 


\section{ORIGINAL ARTICLE}

In the present study gender-wise prevalence of clinical isolates shows that infections caused by Pseudomonas aeruginosa are more common in males (56\%) as compared to females (44\%). This is comparable with study of Rakesk et $\mathrm{al}^{8}$, Javia et $\mathrm{al}^{9}$. The gender wise distribution of patients diagnosed with infections followed the natural epidemiological pattern.

In the present study, the maximum clinical isolates of Pseudomonas aeruginosa were isolated from pus/swab (27\%), followed by urine (19\%). These results are in accordance with other studies $^{8-9}$. The prevalence of the P. aeruginosa isolates varied with the clinical conditions and the samples.

Ceftazidime are the commonest third generation antibiotics in hospital protocols. Resistance to $3^{\text {rd }}$ generation cephalosporin was significant in our study (55\%). These high values of resistance which were observed were higher or comparable with other studies in India ${ }^{7-8}$. The increased prevalence of ceftazidime resistant P. aeruginosa is related to the increased use of beta lactam antibiotics such as amoxicillin and ceftazidime. Selective pressure from the use of antimicrobial agents is a major determinant for the emergence of resistant strains ${ }^{7}$.

Pseudomonas aeruginosa exhibited significant resistance to aminoglycosides. In our study, the rate of aminoglycoside resistance to amikacin and gentamicin was $38 \%$ and $51 \%$ respectively. Thus anti-pseudomonadal effect of amikacin is higher than gentamicin. Consistent with these findings, resistance of Pseudomonas aeruginosa to amikacin was still lower than to gentamicin and this correlates with earlier reports published from India9-11. So, among the aminoglycosides, amikacin has the highest sensitivity and seems to be a promising therapy for pseudomonas infection. Hence, its use should be restricted to only severe nosocomial infections ${ }^{12}$.

The resistance of Pseudomonas to the antibiotics in the quinolone group is not consistent and variability has been reported in different centers ${ }^{8,9}$. In our study, resistance rates against ciprofloxacin and levofloxacin was $63 \%$ and $56 \%$ respectively. Quinolone resistance in our study is high which is comparable to study done by Adhikari L et al ${ }^{13}$.

Resistance rates against imipenem (29\%) and meropenem (27\%) do not show much difference and resistance rate is higher compared to other studies 8,11 . This is because of irrational approach of the clinicians of putting patients on carbepenem therapy straightway without going for antibiotic sensitivity.

Overall we have observed that there is increased antibiotic resistance which may be due to the selective pressure from the use of antimicrobial agents and is a major determinant for the emergence of resistant strains ${ }^{11,14}$.

This study shows that the clinical isolates of Pseudomonas aeruginosa are becoming resistant to commonly used antibiotics and gaining more and more resistance to even newer antibiotics. The antimicrobial agents are losing their efficacy because of the spread of resistant organisms due to indiscriminate use of antibiotics, lack of awareness, patient non-compliance and unhygienic condition.

To prevent the spread of the resistant bacteria, it is critically important to have strict antibiotic policies, while surveillance programmes for multidrug resistant organisms and infection control procedures need to be implemented. In the meantime, it is desirable that the antibiotic susceptibility pattern of bacterial pathogens like Pseudomonas aeruginosa in specialized clinical units be continuously monitored and the results readily made available to clinicians so as to minimize the resistance. 


\section{ORIGINAL ARTICLE}

The solution can be planned by continuous efforts of microbiologists, clinicians, pharmacists and the community to promote greater understanding of this problem. Frequent hand washing to prevent spread of organism should be encouraged. Better surgical and medical care should be provided to patients during hospital stay.

\section{REFERENCES:}

1. Betty A. F., Daniel F. S. and Alice S. W. Bailey \& Scott's Diagnostic Microbiology, Twelth edition, 2007, Pseudomonas, Burkholderia and similar organisms, 340-350, mosby Elsevier, St. Louis, Missouri.

2. Pathmanathan SG, Samat NA, Mohamed R. Antimicrobial susceptibility of clinical isolates of Pseudomonas aeruginosa from a Malaysian Hospital. Malay J Med Sci 2009; 16(2):28-33.

3. Lambert P A. Mechanisms of antibiotic resistance in Pseudomonas aeruginosa. J R Soc Med 2002; 95(suppl 41): 22-26.

4. Babay H A H. Antimicrobial Resistance among Clinical Isolates of Pseudomonas aeruginosa from patients in a Teaching Hospital, Riyadh, Saudi Arabia, 2001-2005. Jpn J Infect. Dis 2007; 60:123-125.

5. Ergin C, Mutlu G. Clinical distribution and antibiotic resistance of Pseudomonas species. Eastern Journal of Medicine 1999; 4(2): 65-69.

6. Holloway WJ, Palmer D. Cinical application of new parenteral antibiotic in treatment of severe bacterial infection. Am J Med 1996; 525-595.

7. K.M. Mohanasoundaram. The Antimicrobial Resistance Pattern in the Clinical Isolates of Pseudomonas aeruginosa in a Tertiary Care Hospital; 2008-2010 (A 3 Year Study). Journal of Clinical and Diagnostic Research. 2011;5(3): 491-494

8. Rakesh M, Govind L, Kalpesh M, Rosy P, Kanu P, Vegad MM. Antibiotic Resistance Pattern In Pseudomonas aeruginosa Species Isolated At A Tertiary Care Hospital, Ahmedabad. National Journal of Medical Research. 2012; 2 (2):156-159

9. Javiya VA, Ghatak SB, Patel KR, Patel JA. Antibiotic susceptibility patterns of Pseudomonas aeruginosa at a tertiary care hospital in Gujarat, India. Indian J Pharmacol 2008; 40:230- 4.

10. Smitha S, Lalitha P, Prajna VN, Srinivasan M, et al. Susceptibility trends of Pseudomonas species from corneal ulcers. Indian J Med Microbiol.2005; 23:168-71.

11. Arora D, Jindal N, Kumar R, Romit. Emerging Antibiotic Resistance In Pseudomonasa Challenge.Int J Pharm Pharm. 2011; 3(2): 82-84

12. Poole K. Aminoglycosides resistance in Pseudomonas aeruginosa. Antimicrob Agents Chem 2005; 49:479-87.

13. Adhikari L, Roy K, Tsering DC, Pal R, Kar S. Susceptibility Rates of Pseudomonas aeruginosa Strains to Quinolones. J. Lab. Physicians.2010;2(2): 121-23

14. Quinn JP. Clinical problems posed by multiresistant nonfermenting gram-negative pathogens. Clin Infect Dis. 1998; 27: 117-4. 


\section{ORIGINAL ARTICLE}

\begin{tabular}{|c|c|c|}
\hline Sex & Total no & Percentage (\%) \\
\hline Male & 85 & 56 \\
\hline Female & 67 & 44 \\
\hline Total & 152 & 100 \\
\hline
\end{tabular}

Table 1: Gender-wise distribution of cases

\begin{tabular}{|c|c|c|}
\hline Name of sample & $\begin{array}{c}\text { No. of Sample in which } \\
\text { Pseudomonas aeruginosa Isolated }\end{array}$ & Percentage (\%) \\
\hline Pus & 41 & 27 \\
\hline Urine & 29 & 19 \\
\hline Endotreacheal Secretion & 24 & 16 \\
\hline BAL & 18 & 12 \\
\hline Sputum & 14 & 11 \\
\hline Blood & 17 & 2 \\
\hline Pleural fluid & 3 & 4 \\
\hline Others & 6 & 2 \\
\hline
\end{tabular}

Table 2: Isolation of Pseudomonas aeruginosa from different clinical samples

\begin{tabular}{|l|c|}
\hline \multicolumn{1}{|c|}{ Antibiotic } & Antibiotic Resistance (\%) \\
\hline Ciprofloxacin & 63 \\
\hline Levofloxacin, & 56 \\
\hline Amikacin & 38 \\
\hline Gentamicin & 51 \\
\hline Tobramycin & 57 \\
\hline Aztreonam & 70 \\
\hline Ceftazidime & 55 \\
\hline Cefepime & 50 \\
\hline Piperacillin & 42 \\
\hline Piperacillin/Tazobactam & 33 \\
\hline Ticarcillin/Tazobactam & 35 \\
\hline Imipenem & 29 \\
\hline Meropenem & 27 \\
\hline Colistin & 12 \\
\hline \multicolumn{2}{r|}{ Table 3: Antibiotic resistance of Pseudomonas aeruginosa } \\
isolated from different clinical samples Antibiotic Resistance (\%) \\
\hline
\end{tabular}




\section{AUTHORS:}

1. Prafulla Songara

2. Sushma Neema

3. Vinita Kothari

4. Ruchi Sachdeva

5. Rupal Deva

\section{PARTICULARS OF CONTRIBUTORS:}

1. Assistant Professor, Department of Microbiolgoy, M.G.M. Medical College, Indore

2. Microbiologist, Cental Lab, Indore.

3. C.E.O., Central Lab, Indore.

4. Quality Manager, Central Lab, Indore.

5. Molecular Microbiologist, Central Lab, Indore

\section{NAME ADDRESS EMAIL ID OF THE} CORRESPONDING AUTHOR:

Dr.Prafulla Songara, Assistant Professor, Department of Microbiology, M.G.M. Medical College, Near SBI Bank, Indore.

Email-drprafulla07@gmail.com

Date of Submission: 13/12/2013.

Date of Peer Review: 14/12/2013.

Date of Acceptance: 23/12/2013.

Date of Publishing: 02/01/2014 